\section{STP: A subroutine for simultaneous test procedures and confidence intervals}

\author{
MARTIJN P. F. BERGER \\ University of Tilburg, The Netherlands
}

After a multivariate analysis of variance has been performed and the overall null hypothesis has been rejected, multivariate multiple comparisons are often required. Only a few multivariate analysis of variance (MANOVA) program packages have an option for simultaneous confidence intervals. These intervals can only be used for minimal hypotheses, which are based on single dependent variables (Roy \& Bose, 1953).

Gabriel $(1968,1969)$ defined a class of simultaneous test procedures (stps) to test all the general linear hypotheses implied by the overall null hypothesis. The class of stps contains procedures based on Roy's largest root, Wilks' likelihood, Hotelling's trace, and Pillai's trace. But Gabriel (1969) proved that, for the general linear hypothesis, the largest-root procedure is the most resolvent one. This means that the largest-root stp will reject every hypothesis that is also rejected by the other stps and possibly more.

The presented subroutine, STP, performs all the tests using the largest-root statistic. Suppose that there are $\mathrm{k}$ levels or groups and $\mathrm{p}$ dependent variables. Then $\left(2^{k}-k-1\right)(2 \mathrm{p}-1)$ simultaneous tests can be made. A $p$ by $p$ hypothesis sums of squares and products (SSCP) matrix $\mathrm{S}_{\mathrm{h}}$ is calculated for each of the $2^{\mathrm{k}}-\mathrm{k}-1$ subsets of the $k$ levels/groups. Corresponding with each of the $2^{p}-1$ subsets of dependent variables, the submatrices $s_{h}$ and $s_{e}$ of $S_{h}$ and the given error SSCP matrix $S_{e}$ can be selected. Finally, the largest-root statistic is computed after solving the eigenstructure of each product $\mathrm{s}_{\mathrm{h}} \mathrm{s}_{\mathrm{e}}^{-1}$. Each largest-root statistic is compared with the common critical value from the largestroot distribution (Heck, 1960).

Description. The simultaneous test statistics and confidence limits are computed by the subroutine STP, which calls the subroutines NROOT and EIGEN (IBM SSP subroutines, 1970), VWRITE (print routine), SSCP (sums of squares and products routine), and COMBI (combination generator).

Input. The subroutine STP can be inserted into any available MANOVA computer program. The dummy arguments of STP must correspond with the error SSCP matrix, the matrix of total scores on the de- pendent variables, the number of levels/groups, the number of dependent variables, and the number of scores per level/group. The largest-root critical value may also correspond with a dummy argument.

Output. The subroutine STP prints the largest-root statistics for the set of general linear hypotheses and confidence intervals on only the minimal hypotheses. The starred values of the statistics exceed the common critical value.

Restrictions. The number of levels or groups and/or the number of dependent variables may not be more than 10 . This restriction can be changed by rewriting the DIMENSION statements. But the amount of computational labor assumes larger proportions as the number of levels and dependent variables increases. The DATA statement in the print routine VWRITE works with REAL array elements holding eight storage characters and INTEGER array elements holding four storage characters.

Computer and Language. All subroutines were written in standard FORTRAN IV and were tested on an ICL 1903T computer. They can, however, easily be converted to other computer systems. The core requirement is approximately $13 \mathrm{~K}$. The total source deck contains 326 cards.

Availability. A listing of the source programs, test data, and output listings can be obtained at no cost from the author: Martijn P. F. Berger, Psychological Laboratory, Hogeschoollaan 225, K. H. Tilburg, Tilburg, The Netherlands.

\section{REFERENCES}

Gabriel, K. R. Simultaneous test procedures in multivariate analys is of variance. Biometrika, 1968, 55, 489-504.

Gabriel, K. R. Simultaneous test procedures: Some theory of multiple comparisons. Annals of Mathematical Statistics, 1969, 40, 221-250.

HECK, D. L. Charts of some upper percentage points of the distribution of the largest characteristic root. Annals of Mathematical Statistics, 1960, 31, 625-642.

IBM 360 Scientific Subroutine Package (360-CM-03X). Programmer's manual (GH20-0205). IBM, 1970.

Roy, S. N., \& Bose, R. C. Simultaneous confidence interval estimation. Annals of Mathematical Statistics, 1953, 24. 513-536. 